\title{
Floor Detection with Indoor Vertical Positioning in LTE Femtocell Networks
}

\author{
José A. del Peral-Rosado*, Michele Bavaro ${ }^{\dagger}$, José A. López-Salcedo*, Gonzalo Seco-Granados*, \\ Pravir Chawdhry ${ }^{\dagger}$, Joaquim Fortuny-Guasch ${ }^{\dagger}$, Paolo Crosta ${ }^{\ddagger}$, Francesca Zanier ${ }^{\ddagger}$ and Massimo Crisci ${ }^{\ddagger}$ \\ *Universitat Autònoma de Barcelona (UAB), Bellaterra, Spain \\ Email: \{JoseAntonio.DelPeral, Jose.Salcedo, Gonzalo.Seco\}@uab.cat \\ $\dagger$ European Commission, Joint Research Center (JRC), Ispra, Italy \\ Email: \{Michele.Bavaro, Pravir.Chawdhry, Joaquim.Fortuny\}@jrc.ec.europa.eu \\ $\ddagger$ European Space Agency (ESA), Noordwijk, The Netherlands \\ Email: \{Paolo.Crosta, Francesca.Zanier, Massimo.Crisci\}@esa.int
}

\begin{abstract}
Vertical positioning is nowadays a topic of high interest in indoor environments, due to the stringent requirements on indoor location accuracy for emergency calls, such as E911. Thus, indoor positioning techniques are investigated to achieve floor detection in order to fulfill these legal mandates. The use of Long Term Evolution (LTE) heterogeneous networks is an attractive solution due to the combination of communications and positioning capabilities. This paper provides an overview of the existing floor detection techniques. Practical results using Global Navigation Satellite Systems (GNSS), inertial sensors, barometer, and LTE signals are obtained in an experimental LTE femtocell network, within a deployment in a two-story building. Probabilities of floor detection above $67 \%$ of the cases are found for the positioning solutions assessed.
\end{abstract}

\section{INTRODUCTION}

Indoor localization is typically achieved by means of specific sensors, dedicated wireless location systems, or existing communication networks [1]. The latter solution is commonly adopted in order to minimize the deployments costs. For instance, wireless local area networks (WLAN) are widely used with fingerprinting methods for indoor positioning, because only received signal strength (RSS) measurements are required. In this sense, cellular networks are usually not foreseen as signals of opportunity for indoor environments due to the severe multipath and attenuation affecting the macro cell signals. However, this trend has changed with the definition of heterogeneous networks in the Long Term Evolution (LTE) standard. The coordination of macro cells with small cells, which are typically located indoors, results in an attractive technology for indoor localization. In addition, LTE specifies dedicated wideband signals [2] and protocols [3] to support location methods, such as the positioning reference signal (PRS) and the LTE positioning protocol (LPP).

The convergence of both communication and positioning capabilities within the LTE system is of special interest for emergency services, as well as location-based services (LBS), which require both features. At the beginning of 2015, the Federal Communications Commission (FCC) approved in [4] new rules on indoor location accuracy of emergency calls, i.e. E911. This legal mandate requires a dispatchable location, or 50 meters of horizontal accuracy for $40 \%$ and $80 \%$ of 911 calls within two and six years, respectively, while providing vertical location [4]. This leads to the need to determine the floor and building location of the mobile user in distress. Thus, the Third Generation Partnership Project (3GPP) consortium is studying indoor positioning enhancements for the LTE standard [5].

The primary method for LTE positioning is based on assisted Global Navigation Satellite Systems (A-GNSS). However, due to the blockage of satellite signals in urban and indoor environments, the standard specifies secondary positioning methods that rely on the cell coverage and signal measurements. As Release 12 of 3GPP LTE, also known as LTE Advanced (LTE-A), these techniques are enhanced cell ID (E-CID), observed time-difference of arrival (OTDoA), uplink time-difference of arrival (UTDoA), and radio frequency pattern matching (RFPM). The scenarios under study include the combination of macro cells with small cells (e.g. femtocells or picocells) in indoor environments, using three-dimensional (3D) channel models [6]. The simulation results reported by 3GPP consortium show the horizontal and vertical positioning accuracy, such as in [7] and [8]. Still, to the best of the authors' knowledge, very few practical results on positioning have been presented with a deployed LTE network based on small cells. Thus, the aim of this paper is to provide an overview of the LTE positioning methods, focusing on their capabilities to achieve floor detection in indoor environments. For this purpose, empirical results are obtained in a experimental LTE femtocell network, based on four base stations (BSs) located in two floors. This practical evaluation includes localization techniques based on GNSS, inertial sensors, barometer and LTE signals, and considers the achievable accuracy, the E911 indoor location requirements, and the implementation cost.

This paper is structured as follows. An overview of the technologies presented in the literature for floor detection is provided in Section II. The indoor positioning techniques available in LTE are evaluated in Section III. Practical results in a real LTE femtocell network are shown in Section IV, before drawing the conclusions in Section V.

\section{LITERATURE SURVEY}

Most of indoor localization solutions are focused on horizontal positioning, even if the height of the reference or mobile devices may have an impact on the location accuracy [9]. This section provides an overview of $3 \mathrm{D}$ or vertical positioning solutions that are already proposed by existing technologies. 


\section{A. Sensors}

Several sensors can be used to determine the altitude of a mobile device in indoors, such as a multi-story building. One of the most popular solutions is to measure the barometric pressure. The use of pressure sensors for indoor navigation has been widely studied, such as in [10] and [11], showing a vertical positioning accuracy in the order of decimeters or sub-meter level. Still, barometric sensors require height thresholds for floor detection, and they are sensitive to local pressure conditions. Thus, many authors combine several types of sensors, such as inertial or magnetometer, in order to obtain more accurate and robust 3D indoor positioning, such as in [12] and [13]. But, this hybrid positioning solution is also more complex, including the calibration of the measurements used.

\section{B. Proprietary technologies}

Dedicated systems are usually designed and implemented to achieve vertical positioning within certain performance requirements, resulting in proprietary technologies. As an example, GNSS pseudolites are added to the GNSS localization solution in order to improve the vertical dilution of precision (VDOP), as it is discussed in [14]. The pseudolites are reference BSs located at low elevations, which can be at rooftop of buildings or in indoors. These BSs retransmit the current GNSS signals in order to improve the signal availability. The GNSS pseudolite solution was demonstrated in [15] to meet some of the E911 indoor location requirements. In general, the approach followed by proprietary solutions is based on the placement of specific beacons in order to achieve 3D indoor positioning with millimeter range accuracy, e.g. based on ultra-wideband (UWB) technology [16], or with acceptable floor detection, e.g. based on Bluetooth technology [17]. Although these specific systems may achieve accurate vertical positioning, their main disadvantage is the deployment cost of the dedicated infrastructure.

\section{Wi-Fi (IEEE 802.11)}

The use of existing WiFi networks, which follow IEEE 802.11 standard, is a widely adopted solution to provide vertical positioning capabilities to mobile devices. Since these devices may be already connected to the network, they do not require any extra equipment, because they typically use RSS measurements. The main approaches for floor determination are either based on the proximity of an access point (AP), whose location is typically known, based on fingerprinting databases, or based on path-loss models. Floor detection probabilities above $90 \%$ are reported in [17] and [18]. However, this achievable performance is strongly affected by the AP availability, the quality of the RSS measurements and the localization technique, which may lead to the use of sensors to avoid outages [19]. In addition, fingerprinting methods require a calibration or wardriving phase, where the RSS measurements are collected, assigned to a specific location, and recorded in a database. Maintenance of the database is also required to ensure its reliability. Still, the computational complexity of floor determination using WiFi fingerprinting databases can be low, such as in [20].

\section{D. $3 G P P$ LTE}

The deployment of LTE heterogeneous networks is also of special interest for indoor positioning. Most of the vertical positioning techniques are based on E-CID methods [21] and RFPM or fingerprinting algorithms by using RSS measurements [22]. Simulation results in [7] and [8] report a vertical accuracy below 3 meters in scenarios with indoor small cells. These results show that E-CID might be adequate for accurate floor determination in indoor deployments, in contrast to OTDoA techniques that may not provide any benefit for vertical positioning. Thus, the use of LTE networks can be specially convenient in order to fulfill E911 indoor location requirements [4], without implying an extra cost on the network deployment. Still, further study of the LTE positioning techniques is required in indoor environments.

\section{E. Hybrid solutions}

The most promising approach is the combination of multiple positioning technologies. Hybrid solutions improve horizontal and vertical localization performance by fusing measurements from different systems. For instance, this can be achieved with simultaneous localization and mapping (SLAM) techniques, such as in [23], [24] and [25]. Any type of information can be included in these algorithms, such as RSS and ranging measurements, sensor and proximity data, floor plans or maps. Cooperative localization algorithms can also be adopted in order to take advantage of different peers, such as in a wireless sensor network (WSN).

\section{IndoOR Positioning Methods}

Despite of the advanced features of current mobile devices, there are several issues, such as computational burden and energy consumption, that limit the adoption of most of the positioning technologies described in the previous section. Thus, this section describes only those indoor positioning methods specified (or under study) in the LTE standard. The main advantages and disadvantages of each method applied to floor detection are summarized, considering also their application to legacy mobile devices.

\section{A. Barometric pressure}

Pressure measurements can be obtained at the user equipment (UE) by using built-in barometers. The altitude $h$ can be estimated with the atmospheric pressure $P$ as [26]

$$
h=44330 \cdot\left(1-\frac{P}{P_{0}}\right)^{-5.255},
$$

where $P_{0}$ is the pressure at sea level, e.g. $1013.25 \mathrm{hPa}$. Barometric sensors are already embedded in many mobile devices, providing a good altitude accuracy. Thus, this is a candidate positioning solution to achieve floor detection in most of the cases. However, legacy devices may not have included theses sensors. In addition, barometer measurements have several sources of error [27], such as humidity, temperature, sea level barometric drift, climate control system in buildings or quantization errors at the location server. Possible solutions are proposed in [27] based on the inclusion of pressure sensors in reference BSs, or the provision of assistance data for sensor calibration, which implies an extra implementation cost. 


\section{B. GNSS-based}

The A-GNSS positioning method is based on the assistance data provided by the network in order to improve the time-tofirst-fix (TTFF), as well as to allow the operation of highsensitivity GNSS (HS-GNSS) receivers at very low levels of signal power. However, satellite signal availability is scarce or even null in indoor environments. Thus, inertial navigation systems (INS) are integrated with GNSS receivers, resulting in GNSS/INS positioning methods. These methods require a good initialization of the localization solution, and periodical GNSS signal availability in order to correct the drift of the sensor measurements. Indoor vertical positioning is typically achieved thanks to the $\mathrm{z}$-axis accelerometer. Another solution is the use of GNSS pseudolites, which is proposed for evaluation in LTE [5] within the study on terrestrial beacon systems (TBS). These GNSS-based positioning methods can accurately determine the floor location, but they require specific network and user equipment.

\section{Cell coverage}

Floor detection can be achieved based on the cell coverage or proximity of a BS, by means of E-CID methods. These are low-cost and robust positioning methods that network operators have extensively used. However, the vertical accuracy of E-CID methods is typically coarse, and depends on the cell radius. In LTE, heterogeneous networks are classified by macro cells and small cells, which are formed by microcells, picocells and femtocells (i.e. smallest BSs). Still, dense network deployments operating at the same frequency band may decrease the E-CID positioning accuracy due to interference. Complementary signal measurements, such as time advance (TA) or angle-of-arrival (AoA), can improve the location performance.

\section{TDoA-based}

LTE defines two TDoA-based positioning methods, i.e. OTDoA and UTDoA, for the downlink and uplink transmission modes, respectively. According to [3], the UE or the network performs ranging measurements and the location server computes the UE position by means of a trilateration technique. Although these methods may achieve acceptable horizontal accuracy in indoors, there are two main limitations on the use of TDoA-based positioning methods for floor detection. First, the typical LTE indoor deployments are based on asynchronous heterogeneous networks, such as femtocells. In these scenarios, the location server cannot directly obtain the UE position solution from the UE-based (i.e. OTDoA) or network-based (i.e. UTDoA) ranging measurements. Thus, synchronization protocols or methods should be used in order to remove the clock offsets between the start of the radio frame of each BS. Second, BSs are located at a similar height, resulting in a very bad geometry for vertical localization. Therefore, TDoAbased methods are not considered as a primary method for floor detection [28]. In addition, interference coordination and multipath mitigation techniques may be needed in order to reduce the positioning errors.

The achievable TDoA accuracy in indoor femtocell networks can be assessed with the Cramér-Rao bound (CRB).
However, the precise characterization of this harsh environment should include clock offsets, shadowing, multipath, nonline-of-sight (NLoS) conditions, and interferences. A simplified model can be considered in order to just determine the feasibility of a certain vertical accuracy in the indoor femtocell scenario. Let us define the range difference between user position, $\mathbf{x}=(x, y, z)^{T}$, and the position of the most powerful $L$ BSs, $\mathbf{x}_{i}=\left(x_{i}, y_{i}, z_{i}\right)^{T}$ for $i=1, \ldots, L$, as

$$
\mathbf{d}=\left|\mathbf{x}-\mathbf{x}_{1}\right|-\left|\mathbf{x}-\mathbf{x}_{j}\right|, \quad j=2, \ldots, L .
$$

Then, the range difference estimates are expressed as

$$
\hat{\mathbf{d}}=\mathbf{d}+\mathbf{n}, \quad \mathbf{n} \sim \mathcal{N}(0, \mathbf{R}),
$$

where $\mathbf{n}$ is the noise vector assumed to be additive white Gaussian noise (AWGN) with constant covariance matrix $\mathbf{R}$,

$$
\mathbf{R}=\left(\begin{array}{cccc}
\sigma_{1}^{2}+\sigma_{2}^{2} & \sigma_{1}^{2} & \cdots & \sigma_{1}^{2} \\
\sigma_{1}^{2} & \sigma_{1}^{2}+\sigma_{3}^{2} & \cdots & \sigma_{1}^{2} \\
\vdots & \vdots & \ddots & \vdots \\
\sigma_{1}^{2} & \sigma_{1}^{2} & \cdots & \sigma_{1}^{2}+\sigma_{L}^{2}
\end{array}\right)
$$

being $\sigma_{i}$ the standard deviation. The achievable ranging accuracy using LTE PRS ${ }^{1}$ is computed with the CRB for time-delay estimation of BS $i$ as [29]

$$
\sigma_{i}^{2} \geq \operatorname{CRB}_{i}(\tau)=\frac{T_{s}^{2}}{8 \pi^{2} \cdot \mathrm{SNR}_{i} \cdot \sum_{k \in \mathcal{K}} \sum_{n \in \mathcal{N}} p(k, n)^{2} \cdot k^{2}},
$$

where $T_{s}$ is the symbol period, $\mathrm{SNR}_{i}$ is the signal-to-noise ratio (SNR) of BS $i, \mathcal{K}$ is the set of PRS symbols, $\mathcal{N}$ is the set of PRS subcarriers, and $p(k, n)^{2}$ is the relative power weight at symbol $k$ and subcarrier $n$. Considering the derivation in [30], the CRB for 3D TDoA localization results in

$$
\operatorname{CRB}(\mathbf{x})=\left(\mathbf{D}^{T} \mathbf{R}^{-1} \mathbf{D}\right)^{-1},
$$

where the Jacobian matrix of $\mathbf{x}$ is

$$
\mathbf{D}=\left(\begin{array}{ccc}
\frac{x-x_{1}}{d_{1}}-\frac{x-x_{2}}{d_{2}} & \frac{y-y_{1}}{d_{1}}-\frac{y-y_{2}}{d_{2}} & \frac{z-z_{1}}{d_{1}}-\frac{z-z_{2}}{d_{2}} \\
\frac{x}{d_{3}} & \frac{y-y_{1}}{d_{1}}-\frac{y-y_{3}}{d_{3}} & \frac{z-z_{1}}{d_{1}}-\frac{z-z_{3}}{d_{3}} \\
\vdots & \vdots & \\
\frac{x-x_{1}}{d_{1}}-\frac{x-x_{L}}{d_{L}} & \frac{y-y_{1}}{d_{1}}-\frac{y-y_{L}}{d_{L}} & \frac{z-z_{1}}{d_{1}}-\frac{z-z_{L}}{d_{L}}
\end{array}\right) .
$$

The geometric quality of the vertical localization is assessed with the VDOP [31], which is computed as

$$
\mathrm{VDOP}=\sqrt{\sigma_{z}^{2}}
$$

by using the covariance matrix of $\mathbf{x}$

$$
\boldsymbol{\Sigma}_{\mathbf{x}}=\left(\mathbf{D}^{\mathrm{T}} \mathbf{D}\right)^{-1}=\left(\begin{array}{ccc}
\sigma_{x}^{2} & \sigma_{x y} & \sigma_{x z} \\
\sigma_{x y} & \sigma_{y}^{2} & \sigma_{y z} \\
\sigma_{x z} & \sigma_{y z} & \sigma_{z}^{2}
\end{array}\right)
$$

\footnotetext{
${ }^{1}$ Also applicable to the cell-specific reference signal (CRS).
} 


\section{E. RFPM or RF fingerprinting}

Signal measurements can be compared with a reference database in order to achieve indoor positioning by means of RFPM or RF fingerprinting. For instance, LTE signals can be used to compute RSS, TDoA or AoA measurements. These measurements are first assigned to a specific known location, which is recorded in a database. Fingerprinting localization techniques are then used to match reference and UE-based measurements, which drive the vertical localization accuracy. This positioning method can be enhanced with cooperative algorithms, such as in [32]. RFPM can also be extended to new LTE capabilities (still under study), such as device-todevice (D2D) communications, or merged with measurements from other systems, such as GNSS or WiFi.

\section{Practical Results}

An experimental LTE femtocell network is used to obtain practical results on indoor localization. The feasibility of TDoA-based methods is first discussed in this section. Then, the floor detection performance is assessed by considering a barometer, GNSS/INS receiver and the LTE cell ID method, i.e. without using additional RSS or ranging estimates.

\section{A. Experimental LTE femtocell testbed}

The experimental testbed is formed by four LTE femtocell BSs placed in different parts of a two-story building, which is located at the Joint Research Center (JRC) in Ispra (Italy). As it is shown in Figure 1 and 2, two LTE femtocell BSs are installed in each floor. The height between floor levels is around 3.24 meters, and the ground of the first floor is considered the z-axis reference of the local coordinate system. The femtocells are deployed by using a universal software radio peripheral (USRP) controlled by a computer running the LTE software eNodeB of Amarisoft. The USRP N210 model is equipped with SBX transceiver daughterboard and a GPS disciplined oscillator (GPSDO). The transmission of low-power signals is authorized by the Italian Ministry at band 7, i.e. system bandwidth of $10 \mathrm{MHz}$, carrier center frequency of $2625 \mathrm{MHz}$ and E-UTRA absolute radio frequency channel number (EARFCN) equal to 2800. Each BS uses the same software configuration (e.g. $30 \mathrm{~dB}$ of USRP transmission gain) with a different physical cell ID. The network of BSs is not synchronized, but the GPSDO ensures a precise time and frequency stability. Omnidirectional antennas ${ }^{2}$ are located close to the windows at a height of 1.2 meters above the ground of the corresponding floor.

The measurement equipment is based on three units, which are carried with a backpack. First, a GNSS/INS receiver, i.e. Novatel SPAN-CPT, computes a position fix every $200 \mathrm{~ms}$ using integrated gyroscopes and accelerometers, and the Novatel GPS-702 pinwheel antenna. Second, the Bosch BMP180 pressure sensor [26], which is built-in in many smart phones, outputs altitude measurements every second, taking as a reference the clock of a HS-GNSS u-blox receiver. Third, LTE signals are captured with the Tektronix RSA306 spectrum analyzer and the HackRF One, which is a reconfigurable RF front-end or software-defined radio (SDR). In order to improve

\footnotetext{
${ }^{2}$ Antenna model Cobham XPO2V-0.8-6.0-GF/1441 for BS 1 and BS 2, and antenna model Cobham XPO2V-0.8-6.0/1485 for BS 3 and BS 4 .
}

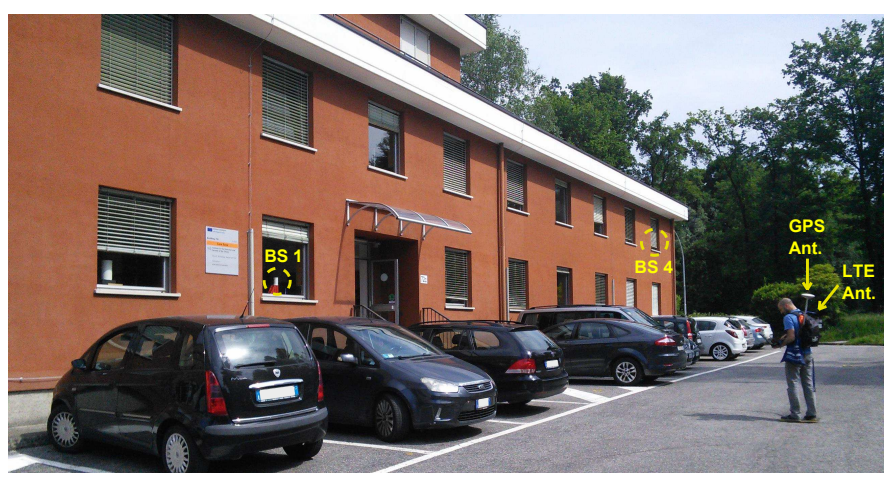

Fig. 1. Experimental LTE femtocell testbed formed by four BSs located in a two-story building of the Joint Research Center (JRC) in Ispra (Italy).

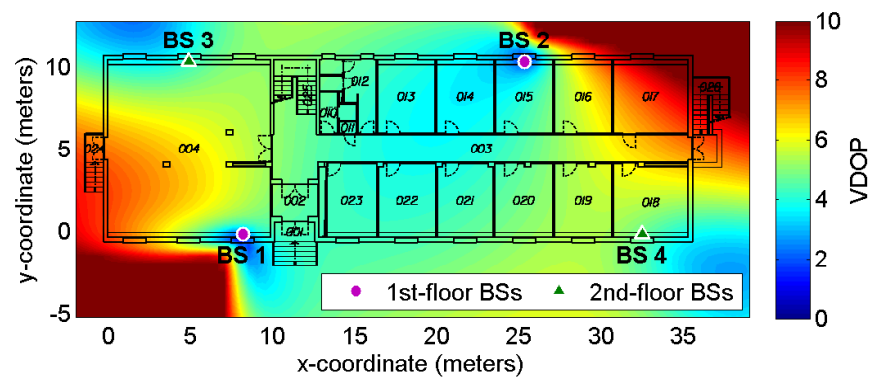

(a) First floor

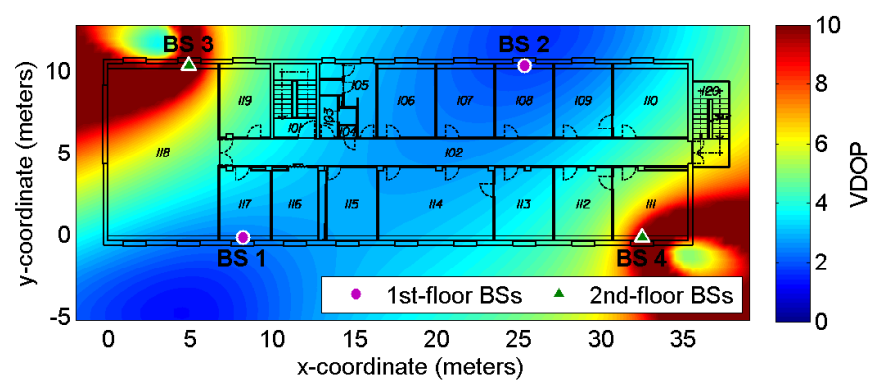

(b) Second floor

Fig. 2. Floor plans and VDOP for TDoA-based estimates in the experimental LTE femtocell testbed.

the signal reception, a low-noise amplifier and a high-pass filter are used after the omnidirectional OmniLOG 70600 antenna. The sampling frequency of the HackRF One is set to 12.5 Msps. The reference height (with respect to the ground) of the three units is $1.85,1.6$ and 1.3 meters, respectively. The equipment is controlled by a laptop, where the measurements are recorded. The data collected is then post-processed with specific software. In particular, the LTE RF baseband samples captured by the SDR are acquired and tracked in snapshots by a MATLAB-based software receiver [33].

Using this experimental testbed, the test scenario is defined by a user walking from outside to inside of the building, and between first and second floor. In order to calibrate the equipment, the user starts at a static outdoor position in the car park, which is at 0.9 meters below the first floor. Then, the user enters the building, goes up directly to the second floor, and walks through the main corridor. Immediately after, this path is repeated in the first floor. Stops of approximately ten seconds are periodically included in this user path. 



Fig. 3. Average SNR estimated using acquired 10-MHz LTE signals along the user trajectory in the experimental femtocell testbed, and z-axis accuracy calculated using one PRS symbol with the CRB for 3D TDoA localization.

\section{B. Feasibility of TDoA for floor detection}

As in typical femtocell deployments, the applicability of TDoA-based methods for indoor vertical positioning is limited by the synchronization of the network and the location of the BSs. The feasibility of TDoA positioning for floor detection is here discussed considering synchronized femto BSs. For this purpose, the VDOP is computed using (8) for the first and second floor of the experimental testbed, and assuming a receiver antenna height of 1.5 meters above the ground. As it is shown in Figure 2, the VDOP values in the main corridor are between 2 and 4, which corresponds to an acceptable geometry. The LTE software receiver in [33] is then used to acquire and track the 10-MHz LTE signals transmitted by each BS of the testbed. Given the user trajectory described in the previous section, the SNR is estimated and averaged every second using the CRS symbols. These SNR measurements are used to compute the CRB for time-delay estimation in (5), and in turn the covariance matrix $\mathbf{R}$ in (4). The achievable z-axis accuracy is finally computed by taking $\sqrt{[\mathrm{CRB}(\mathrm{x})]_{3,3}}$ from (6). The SNR estimates and the vertical accuracy are shown in Figure 3. Given the wide bandwidth of the signal, the achievable vertical accuracy is below 1 meter, which results in a good floor detection. However, multipath and synchronization errors may severely impact this result. Future work is aimed to provide experimental 3D TDoA localization results using LTE signals.

\section{Experimental results}

This section assesses the floor detection performance of indoor positioning methods based on the barometric pressure, GNSS/INS and LTE cell ID. Although these methods can already be implemented in off-the-shelf mobile devices, precise equipment is used to assess the experimental testbed.

The height results are shown in Figure 4(a). The reference is based on the expected height of the user, without considering body oscillations. This reference and the estimates are calibrated to the height of the backpack, i.e. 1.5 meters

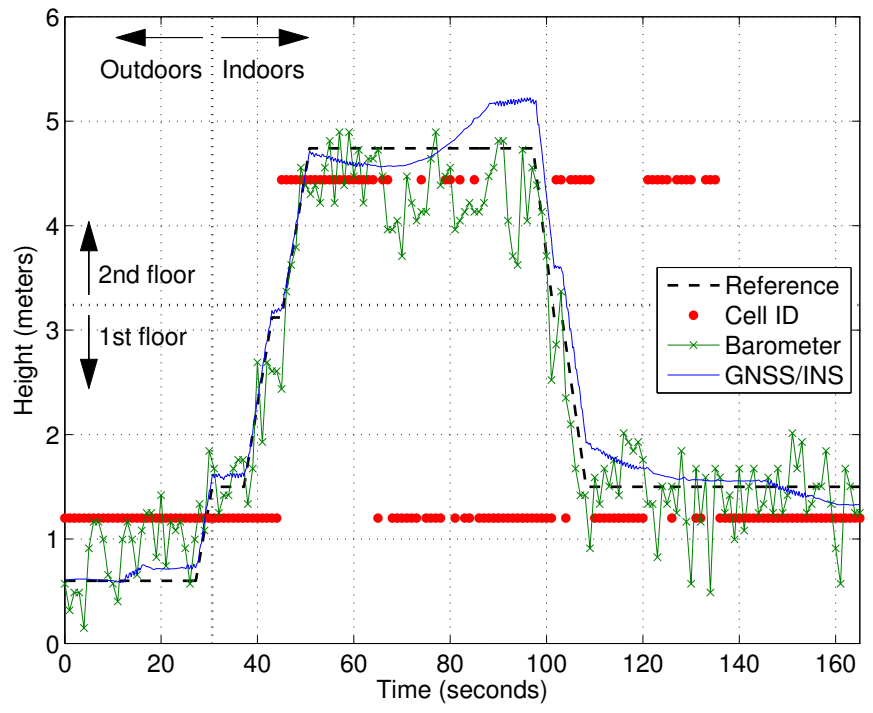

(a) Reference and estimated height

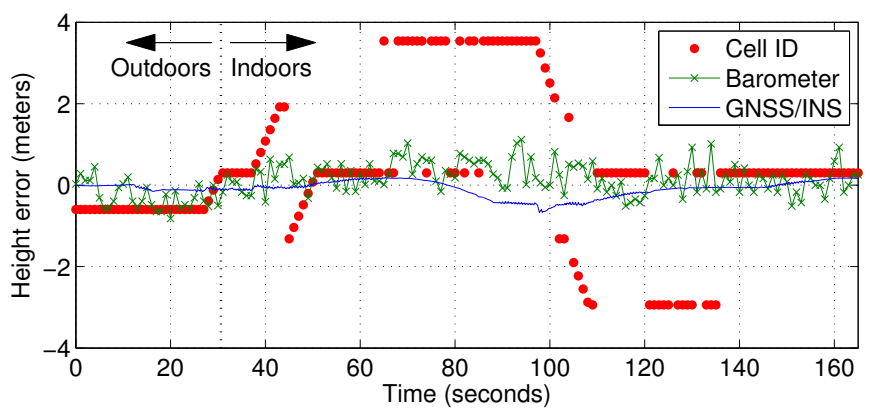

(b) Height error of the estimation

Fig. 4. Height results using positioning methods based on barometric pressure, GNSS/INS and LTE cell ID along the user trajectory.

above the ground. The cell ID method provides only two values, because it is based on the height of the BS acquired (from a set of known IDs). Its performance could be notably improved with RSS or ranging estimates, such as in E-CID. The barometric measurements follow the reference height with an almost constant standard deviation. The z-axis of the GNSS/INS position solution tracks smoothly the vertical trajectory of the user. Floor detection is then achieved based on a threshold level, which is equal to the height level between first and second floor. The errors of these solutions with respect to the reference can be seen in Figure 4(b). The cell ID method varies depending on the signal strength of each BS, which is highly affected by interference and fading events due to the thickness of walls and the floor. The barometric measurements have some height fluctuations that may be produced by small pressure changes. Finally, the GNSS/INS localization accuracy is driven by the drift of the sensors and the satellite signal availability. In order to reduce these drifts, the test has been initialized outdoors in open-sky conditions.

The root mean square error of the height estimates and the floor detection probability are computed for each positioning method. As it is shown in Table I, all of the positioning methods assessed fulfill a tentative E911 requirement of $67 \%$ of floor detection. The barometer and GNSS/INS solutions detect the floor level with a probability higher than $98 \%$. 
TABLE I. FLOOR DETECTION RESULTS IN AN LTE INDOOR TESTBED.

\begin{tabular}{ccc}
\hline Positioning method & Vertical accuracy $(\mathrm{m})$ & Floor detection $(\%)$ \\
\hline Cell ID & 1.79 & 69.88 \\
GNSS/INS & 0.21 & 98.19 \\
Barometer & 0.42 & 98.80 \\
\hline
\end{tabular}

The GNSS-based approach achieves the best vertical accuracy, however it is expected to dramatically decrease its accuracy without a calibration of the INS. The cell ID performance can be improved with a robust acquisition and additional signal measurements, such as RSS and ranging. Thus, the results show the barometric sensor as a robust and reliable solution, which may require punctual calibrations in order to remove fluctuations due to local pressure changes. The cell ID method is then considered a complementary solution. The assessment of other indoor vertical positioning techniques based on TDoA, RFPM or hybrid methods is left for future work.

\section{CONCLUSION}

This paper provides an overview of the positioning technologies and methods for floor detection in indoor environments. An experimental Long Term Evolution (LTE) femtocell network is deployed, and empirical results on vertical localization are provided with a barometer, GNSS/INS receiver and LTE software receiver. This experimental testbed is also used to validate the feasibility of TDoA methods for floor detection in multi-story buildings. Finally, E911 requirement for indoor vertical location is fulfilled with the solutions assessed. Probabilities of floor detection above $90 \%$ have been found with the barometer and GNSS/INS receiver, while the cell ID method using 10-MHz LTE signals has only reached $70 \%$, approximately. Both barometer and LTE cell ID are expected to be the main techniques for floor detection. Evaluation of E-CID, TDoA-based, RFPM and hybrid indoor positioning methods in LTE are left for future work.

\section{ACKNOWLEDGMENT}

The content of the present article reflects solely the authors view and by no means represents the official European Space Agency (ESA) and European Commission (EC) view. This work was supported by the ESA under the NPI programme No. 4000110780/14/NL/AK, and by the Spanish Ministry of Economy and Competitiveness project TEC2014-53656-R.

\section{REFERENCES}

[1] H. Liu, H. Darabi, P. Banerjee, and J. Liu, "Survey of wireless indoor positioning techniques and systems," IEEE Trans. on Systems, Man, and Cybernetics, Part C: Applications and Reviews, vol. 37, no. 6, pp. 1067-1080, Nov. 2007.

[2] 3GPP TS 36.211, Physical channels and modulation, Std., Rel. 9, 2010.

[3] 3GPP TS 36.305, E-UTRAN; Stage 2 functional specification of UE positioning in E-UTRAN, Std., Rel. 9, 2010.

[4] FCC, "Fourth report and order on wireless E911 location accuracy requirements," Tech. Rep., Jan. 2015.

[5] RP-141003, "New SID: Study on indoor positioning enhancements for UTRA and LTE," 3GPP, NextNav, TSG RAN, Jun. 2014.

[6] 3GPP TR 36.873, E-UTRA; Study on 3D channel model for LTE, Std., Rel. 12, 2015.

[7] R1-152634, "Summary of system level analysis for indoor user positioning," 3GPP, Intel Corporation, TSG RAN WG1, May 2015.
[8] R1-152799, "Indoor positioning simulation results," 3GPP, Qualcomm Incorporated, TSG RAN WG1, May 2015.

[9] Y. Zhao, Y. Yang, and M. Kyas, "2D geometrical performance for localization algorithms from 3D perspective," in Proc. IPIN, Oct. 2013.

[10] B. Li, B. Harvey, and T. Gallagher, "Using barometers to determine the height for indoor positioning," in Proc. IPIN, Oct. 2013.

[11] H. Xia, X. Wang, Y. Qiao, J. Jian, and Y. Chang, "Using multiple barometers to detect the floor location of smart phones with built-in barometric sensors for indoor positioning," Sensors, vol. 15, no. 4, pp. 7857-7877, Mar. 2015.

[12] M. Romanovas, V. Goridko, A. Al-Jawad, M. Schwaab, M. Traechtler, L. Klingbeil, and Y. Manoli, "A study on indoor pedestrian localization algorithms with foot-mounted sensors," in Proc. IPIN, Nov. 2012.

[13] W. Song, J. W. Lee, B. S. Lee, and H. Schulzrinne, "Finding 9-1-1 callers in tall buildings," in Proc. IEEE WoWMoM, Jun. 2014.

[14] Y. Liu and S. Tian, "Vertical positioning technologies and its application of pseudolites augmentation," in Proc. WiCOM, Oct. 2008.

[15] CSRIC III Working Group 3, "E9-1-1 location accuracy: Indoor location test bed report," Final Report, Mar. 2013.

[16] C. Zhang, M. Kuhn, B. Merkl, A. Fathy, and M. Mahfouz, "Real-time noncoherent UWB positioning radar with millimeter range accuracy: Theory and experiment," IEEE Trans. on Microwave Theory and Techniques, vol. 58, no. 1, pp. 9-20, Jan. 2010.

[17] E. S. Lohan, J. Talvitie, P. Figueiredo e Silva, H. Nurminen, S. AliLoytty, and R. Pich, "Received signal strength models for WLAN and BLE-based indoor positioning in multi-floor buildings," in Proc. ICLGNSS, Jun. 2015.

[18] R. S. Campos, L. Lovisolo, and M. L. R. de Campos, "Wi-Fi multifloor indoor positioning considering architectural aspects and controlled computational complexity," Expert systems with applications, vol. 41, no. 14, pp. 6211-6223, Oct. 2014.

[19] P. Gupta, S. Bharadwaj, S. Ramakrishnan, and J. Balakrishnan, "Robust floor determination for indoor positioning," in Proc. NCC, Feb. 2014.

[20] M. Abd Rahman, M. Dashti, and J. Zhang, "Floor determination for positioning in multi-story building," in Proc. WCNC, Apr. 2014.

[21] T. Wigren, "LTE fingerprinting localization with altitude," in Proc. IEEE VTC Fall, Sep. 2012.

[22] Y.-h. Wang, H. Li, X.-1. Luo, Q.-m. Sun, and J.-n. Liu, "A 3D fingerprinting positioning method based on cellular networks," International Journal of Distributed Sensor Networks, vol. 2014, Jul. 2014.

[23] R. Faragher and R. Harle, "SmartSLAM-an efficient smartphone indoor positioning system exploiting machine learning and opportunistic sensing," in Proc. ION GNSS+, Sep. 2013.

[24] P. Mirowski, T. K. Ho, S. Yi, and M. Macdonald, "SignalSLAM: Simultaneous localization and mapping with mixed WiFi, Bluetooth, LTE and magnetic signals," in Proc. IPIN, Oct. 2013.

[25] M. Hardegger, D. Roggen, and G. Tröster, "3D ActionSLAM: wearable person tracking in multi-floor environments," Personal and Ubiquitous Computing, vol. 19, no. 1, pp. 123-141, Jan. 2014.

[26] Bosch Sensortec, "BMP180, Digital pressure sensor," Datasheet, 2015.

[27] R1-153224, "Discussion on barometer technology for indoor positioning," 3GPP, Huawei, HiSilicon, TSG RAN WG1, May 2015.

[28] R4-142782, "Indoor and vertical positioning," 3GPP, Alcatel-Lucent, TSG RAN WG4, May 2014.

[29] J. A. Del Peral-Rosado, J. A. López-Salcedo, G. Seco-Granados, F. Zanier, and M. Crisci, "Achievable localization performance accuracy of the positioning reference signal of 3GPP LTE," in Proc. ICL-GNSS, Jun. 2012.

[30] R. Kaune, J. Hörst, and W. Koch, "Accuracy analysis for TDOA localization in sensor networks," in Proc. FUSION, Jul. 2011.

[31] K. Borre et al., A software-defined GPS and Galileo receiver: a singlefrequency approach. Birkhauser, 2007.

[32] R. M. Vaghefi and R. M. Buehrer, "Cooperative RF pattern matching positioning for LTE cellular systems," in Proc. PIMRC, Sep. 2014.

[33] J. A. del Peral-Rosado, J. M. Parro-Jiménez, J. A. López-Salcedo, G. Seco-Granados, P. Crosta, F. Zanier, and M. Crisci, "Comparative results analysis on positioning with real LTE signals and low-cost hardware platforms," in Proc. NAVITEC, Dec. 2014. 